\title{
A preferential looking clinical acuity test: Improvements in implemented microcomputer control
}

\author{
LEE E. OSTRANDER and GILLRAY L. KANDEL \\ Rensselaer Polytechnic Institute, Troy, New York \\ and \\ PAUL L. JENKINS and JOHN W. SIMON \\ Albany Medical College, Albany, New York
}

\begin{abstract}
A manually operated preferential looking grating acuity test was modified to include a microcomputer for controlling both stimulus presentation and data acquisition, storage, and analysis. The computerized test requires only one observer-operator and minimizes several testing biases and errors of the manual test. A technique was developed to keep the observer-operator masked during the generation of a transformed staircase. The monocular test time of about $8 \mathrm{~min}$, and the success rate- $81 \%$ for 224 children aged 2-39 months-compare favorably with our experience with the manual two-operator test. For 39 pairs of age-matched children without visual pathology, grating acuities were 0.6 octaves better with the computerized test. The computerized version was used to measure acuity in 120 children who had, according to clinical examination, poorer vision in one eye than in the other. The suspect eyes showed an average of 0.8 octaves poorer grating acuity. The improvements of computerization were realized without loss of the advantages of the manual test.
\end{abstract}

During the last several years, a preferential looking (PL) apparatus similar to that described by Gwiazda, Brill, Mohindra and Held (1978) has been used in the Pediatric Ophthalmology Service of the Albany Medical Center. It has been used in the diagnosis and treatment of more than 800 babies. This grating visual acuity test was first developed some years ago by Gwiazda and her associates for use in visual research with normal infants (Gwiazda et al., 1978; Gwiazda, Brill, Mohindra, \& Held, 1980; Gwiazda, Wolfe, Brill, Mohindra, \& Held, 1980 ). It presents to the child two brightly projected stimuli in a darkened room (Gwiazda et al., 1978), rather than two pictures of gratings (Teller, Morse, Barton, \& Regal, 1974) in a lighted environment. Head and eye movements, in accordance with the pattern preference phenomenon described by Fantz (1958) provide a basis for grating acuity determination.

Our original version required two people to operate it, one to observe the child and the other to operate the projectors and record responses. Although it has served useful clinical purposes (Catalano, Simon, Jenkins, \& Kandel, 1987; Jenkins, Simon, Kandel, \& Forester,

This work was supported by NEI Grant EY05816 to John W. Simon. Requests for reprints, circuit diagram, and computer source code may be sent to Gillray L. Kandel, Psychology Department, Rensselaer Polytechnic Institute, Troy, NY 12180-3590.
1985), we wanted to minimize possible measurement bias, recording errors, and other inaccuracies that we detected in its use. Accordingly, the apparatus has been adapted for microcomputer control of stimulus presentation and of data acquisition, storage, and analysis, so that the test now requires only one observer-operator.

\section{APPARATUS}

The instrument includes a panel containing the observer's viewing ports and two rear-projection screens, as is shown in Figure 1. At the child's viewing distance of $51 \mathrm{~cm}$, each of the screens subtends $11^{\circ}$; their centers are $32^{\circ}$ apart. Two Kodak E-2 Ectographic Carousel projectors are located on a platform behind the observer.

Each Carousel tray carries a series of 42 transparencies arranged in 14 triads. Each triad comprises a black and white square-wave stimulus grating (hereafter called grating), a polychromatic cartoon figure (hereafter called cartoon), and a square-wave grating of $72 \mathrm{cpd}$ (hereafter called gray) whose projected space-averaged luminance is equal to that of the projected stimulus grating. ${ }^{1}$ The grating of each triad is paired with a gray in the opposite Carousel, while each cartoon figure is paired with a similar cartoon figure. Selection of the appropriate corresponding Carousel positions within each paired triad 


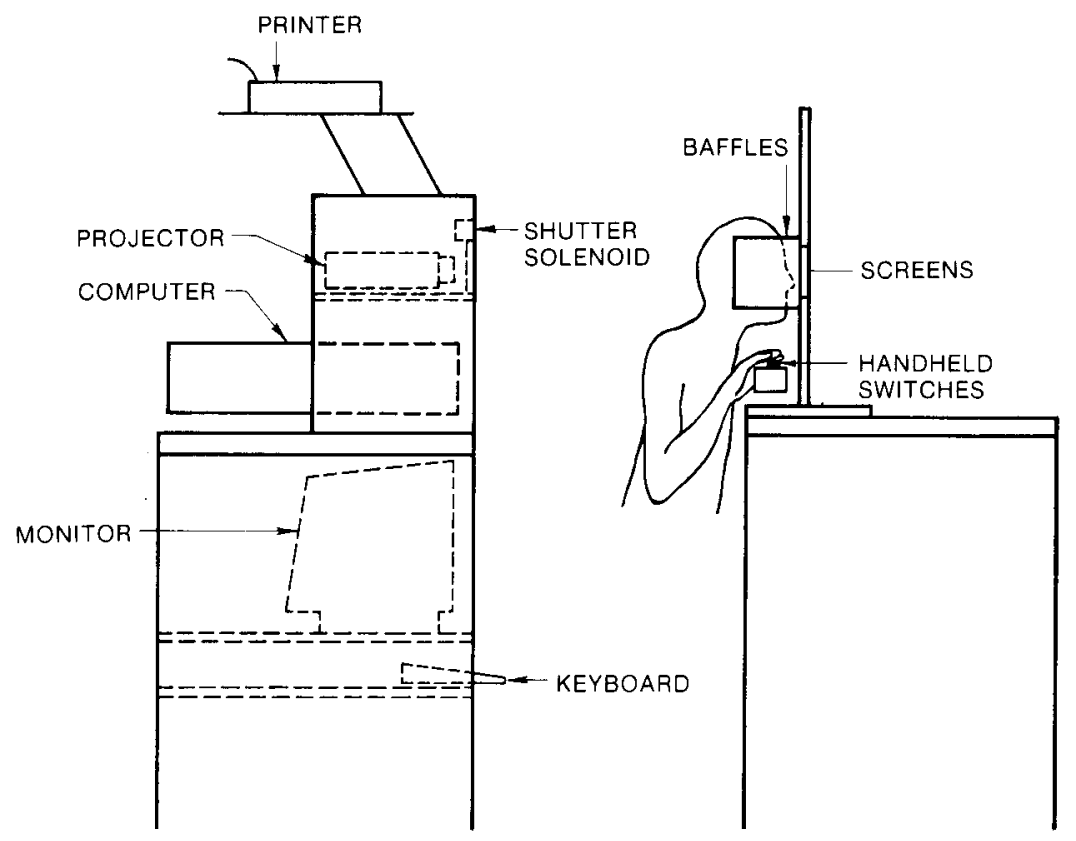

Figure 1. Side view of test apparatus (not to scale). The observer-operator peers at the patient, who is on the other side of the screen, through the baffled viewing ports between the screens. The computer is operated with the hand-held switches. Access to the monitor and keyboard is not required during the test procedure.

delivers a grating image to either the right or the left screen and a gray to the other screen. Alternatively, the matched cartoons can be presented. The gratings in each triad are ordered successively in approximately halfoctave steps from 0.375 to $36 \mathrm{cpd}(20 / 1,600$ to $20 / 17$ Snellen equivalents).

\section{PROCEDURES}

Before projecting a grating from a specific step, the computer, an IBM PC, presents the cartoon pair within that step. It then selects the side on which the grating is to be projected by rotating both Carousels either one position forward or one backward. All subsequent grating presentations are similarly preceded by the cartoon pair of the step to which the grating belongs. The computer is constrained not to project gratings on the same side more than three times in succession. Within this constraint, the grating is projected randomly to the left or right on every test trial. This randomization, together with the presentation of a cartoon pair before each grating-gray pair, keeps the observer masked. The parent, who holds the child during testing, may be asked to wear nonreflecting, semi-opaque glasses, which are adjusted to permit them to see the child but not the screens.

The projectors are computer-controlled by means of a Data Translation input-output board (DT 2805) and a locally constructed switching interface unit that connects the projectors to the computer. The computer's control functions follow the algorithm of Table 1 . The computer synchronously moves both Carousels, in response to the observer-operator's judgments.
Some babies become distressed when the computer advances the Carousels through two steps during Sequence 0,1 , or 2 , identified in Table 1 . Such advances project as many as four monochrome and two cartoon pairs in rapid succession. However, when the projectors' lights are completely extinguished during multiple Carousel position changes, some babies become fussy during the dark period. Therefore an auxiliary system of shutters is mounted in such a way that, when activated by the computer, they attenuate about $85 \%$ of the light from the projectors. These shutters are operated in synchrony with the computer's commands to rotate the Carousels. The resulting system operates the projectors in a random access mode of presentation under computer control.

The observer-operator's judgments as to whether the infant is looking to the right or to the left are entered into the computer by activation of a hand-held unit. In addition to the right-left decision, the operator may indicate an error in the immediately preceding decision. This is signaled by a prolonged depression of the left-right switch. This action results in another presentation at the same step level.

The system software is menu driven, with selections as shown in Figure 2. At the initiation of each test, the Carousels are placed at a preselected index position. Demographic information is then entered in response to questions that appear on the computer screen with the appropriate menu selection. To begin an acuity measurement, the computer first moves the Carousels to the cartoon figures of the triad that is approximately three octaves below the authors' laboratory's grating acuity norm for the child's age. At 2.5 months or younger, $0.375 \mathrm{cpd}$ 
Table 1

Computer Program Decision Algorithm

\begin{tabular}{|c|c|c|c|}
\hline $\begin{array}{l}\text { Current } \\
\text { Sequence }\end{array}$ & $\begin{array}{l}\text { Previous } \\
\text { Response }\end{array}$ & $\begin{array}{l}\text { Current } \\
\text { Response }\end{array}$ & $\begin{array}{l}\text { Computer Programmed Rules; } \\
\text { Action on Next Trial }\end{array}$ \\
\hline 0 & $\mathbf{S}$ & C & Ascend two steps \\
\hline 0 & $\mathbf{S}$ & I & Descend two steps \\
\hline 0 & $\mathrm{C}$ & $\mathbf{C}$ & Ascend two steps \\
\hline 0 & C & I & Begin Sequence 1; use its rules \\
\hline 0 & I & $\mathrm{C}$ & Stay at same step \\
\hline 0 & I & I & Descend two steps \\
\hline 1 & $\mathrm{X}$ & I & Descend two steps \\
\hline 1 & I & C & Stay at same step \\
\hline 1 & $\mathrm{C}$ & C & Begin Sequence 2; use its rules \\
\hline 2 & $\mathrm{X}$ & $\mathrm{C}$ & Ascend two steps if appropriate* \\
\hline 2 & $\mathrm{X}$ & I & Begin Sequence 3 ; use its rules \\
\hline 3 & $\mathrm{X}$ & I & Descend one step \\
\hline 3 & I & $\mathrm{C}$ & Stay at same step \\
\hline 3 & $\mathrm{C}$ & $\mathrm{C}$ & Begin Sequence 4 ; use its rules \\
\hline 4 & $\mathrm{X}$ & $\mathrm{C}$ & Ascend one step if appropriate* \\
\hline 4 & $\mathrm{X}$ & I & Begin Sequence 5 ; use its rules \\
\hline 5 & $\mathrm{X}$ & I & Descend one step \\
\hline 5 & I & $\mathrm{C}$ & Stay at same step \\
\hline 5 & $\mathrm{C}$ & $\mathrm{C}$ & Terminate test \\
\hline
\end{tabular}

Note-Sequence refers to a series of monotonic steps headed either upward or downward. Each is bounded by direction reversals. A trial is a presentation of a gray-grating pair at a given step. A step is one of 14 triads consisting of a gray, a grating, and a cartoon figure. The steps are in ascending order for increased grating frequency. The coding of computer responses or actions is as follows: $\mathbf{S}=$ Start, no test grating exposed. $\mathbf{C}=$ Correct response to grating. $\mathrm{I}=$ Incorrect response to grating. $\mathrm{X}=$ Either a correct or an incorrect response to grating. *An ascent is appropriate if there are two correct responses at the same step; otherwise stay at the same step.

$(20 / 1,600)$ is the starting point. One step higher is selected for age intervals beginning at $6,10,14,20$, and 25 months. For patients 36 months and older, initial presentations are at $6.0 \mathrm{cpd}(20 / 100)$.

The system design required a choice of grating frequencies. The range of grating frequencies selected $(0.375$ to $36 \mathrm{cpd}$ ) accommodates a wide variation in visual acuities. When a child is introduced to the test, the coarser gratings of Sequence 0 (Table 1) first provides the child with an orientation to the test and allows the tester to observe the child's characteristic response patterns in the test situations. Because the starting level is usually well below the child's grating acuity, the algorithm should cause a consistent ascent with eventual convergence on the child's grating resolution. Initial progression up the staircase, although not known until after testing is completed, confirms that the test has likely yielded a valid measure of acuity. If the child's visual acuity is below the starting level, the initial trend on the staircase should be downward. If responses below the lowest step are called for, the computer signals the operator with a message and a warning tone to indicate that the acuity estimate will be biased. Nevertheless, completion of the test proceeds using the lowest step in place of the lower ones demanded by the situation. The requirement for steps below the lowest is apt to arise when a child's visual acuity is below approximately $20 / 1,200$.

The algorithm that controls subsequent presentations (Table 1) produces a transformed staircase (Levitt, 1970;
Rose, Teller, \& Rendleman, 1970) with six reversals. When the test result is called forth by the operator, the computer calculates visual acuity as the geometric mean of the spatial frequencies of the six steps at which the reversals have occurred.

In addition to the child's grating acuity, expressed both in cycles per degree and in Snellen equivalent, this instrument automatically extracts several measures from which one can infer the dependability of a given eye's test result. It calculates an index of lateral response bias for the grating stimuli as well as for the cartoon figures. This indicates the degree to which the infant-operator system responds so as to favor one screen or the other, and thus provides a measure of internal consistency for the entire system. Approximately equal numbers of left and right judgments are expected. If they differ greatly, then the validity of the result can be held to be questionable and gives one pause to consider possible sources of such inconsistency.

The computer measures the time between stimulus presentation and observer-operator response for each test pair and for cartoon figures. It computes the averages of these, as well as the total elapsed test time. These serve as objective measures of the smoothness of the procedure and the alertness of the patient. Finally, the computer also calculates (in octaves) a measure of convergence defined as the standard deviation of the log spatial frequency (in cpd) at reversal points. It is assumed that a smaller variance indicates that the test procedure has more narrowly delineated the child's grating acuity.

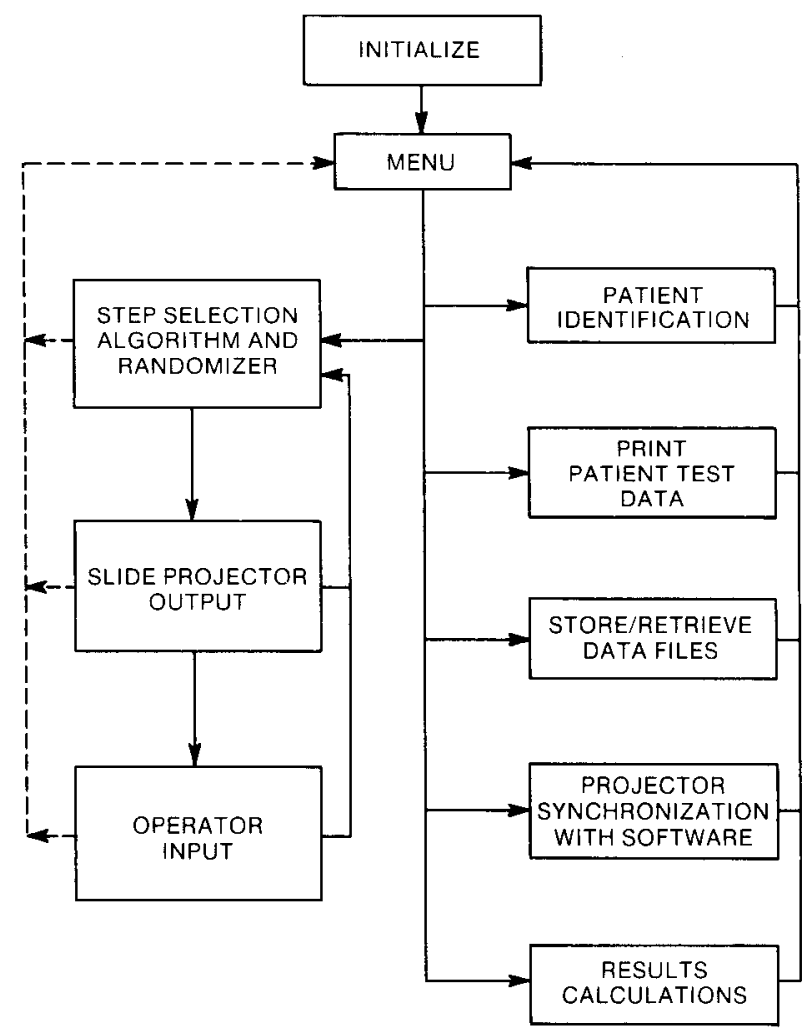

Figure 2. Functional block diagram of computer program. 


\section{RESULTS}

In the first 8 months after microcomputerization we attempted tests on 224 babies, aged 2-39 months. All were referred because of strabismus, amblyopia, or other visual system pathology. Eighty-one percent of these tests were completed so as to yield clinically useful information. This rate, which pertains to successfully completed tests on the first attempt, compares favorably with our $79 \%$ success rate on the first or second attempt using the two-operator manual test. We define clinically useful information as either successful monocular results for both eyes or a successful monocular result in the clinically suspect eye and a successful binocular result.

The mean monocular testing time for the computerized test is about $8 \mathrm{~min}(S D=6 \mathrm{~min})$, so the computerized test is at least as rapid or more rapid than the two-operator manual version. Seventy percent of patients completed the full test protocol of six reversals, taking an average of about 7 min per eye. The tests that were terminated after three to five reversals because of the child's failure to cooperate ( $8 \%$ of patients) required an average of about $13 \mathrm{~min}$ per eye. The average time per judgment, including that for cartoon figures, is about $10 \mathrm{sec}$. Judgments of 18 to 36 grating trials are usually needed to complete one test.

Figure 3 presents, for the computerized test, a regression line for log grating acuity versus log age of children (in months). These monocular acuities are from children without discernible visual system pathology (normals). Summary data obtained from figures published in two other studies that involved a manual test with apparatus similar to the authors' are comparable.

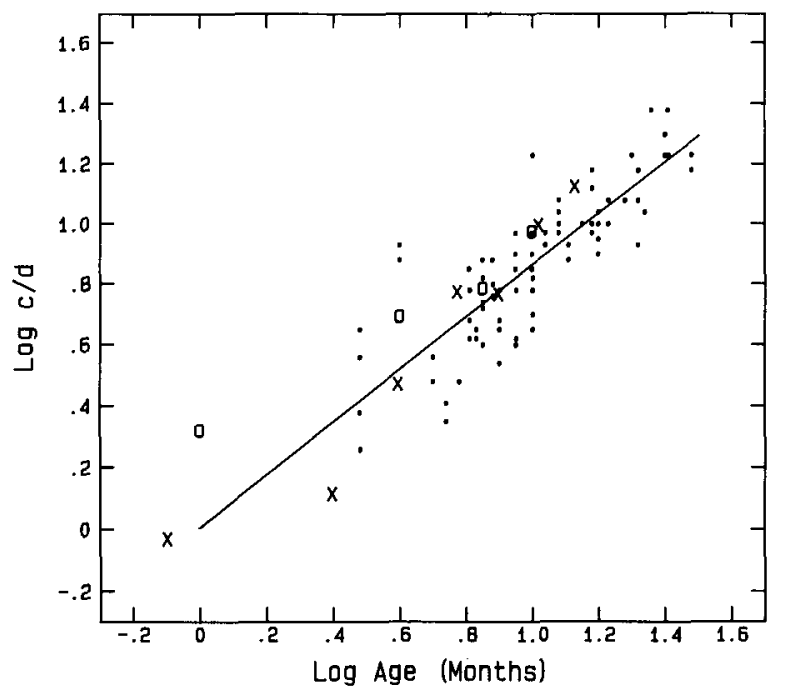

Figure 3. Log grating acuity (monocular) obtained with the computerized test versus log age for 39 babies, 3 to 30 months old, who are without visual pathology. Data indicated by 0 = Birch (1985; monocular) and by $X=$ Gwiazda, Brill, Mohindra, and Held (1980; binocular). The acuities from both of these studies were obtained with two-person manual versions of the test. The line and filled points show the computerized test results of the present study.
The mean grating acuities of 39 normals, aged 3 to 27 months, obtained with the computerized test, were also compared with 39 other age-matched normals tested previously with our manual version of the PL test. Grating acuities measured with the computerized test were about 0.6 octaves higher than those measured with the manual test. This difference is statistically significant $(t=2.14$, $p=.018$ ). In part, this difference can be attributed to the smaller step sizes of the computerized test, which has half-octave steps instead of the full-octave ones that the manual test had.

A group of 120 consecutive patients, aged 2 to 30 months, was identified; they included all those who had successful monocular tests with the computerized version and had one eye that was clinically suspect of having poorer visual function. The diagnoses in this group of patients included strabismus, anisometropia, media opacity, nystagmus, retinoblastoma, and eyelid tumors. The clinically suspect eyes had significantly poorer visual acuities, with a mean difference of 0.8 octaves $(t=2.93$, $p=.004)$.

\section{DISCUSSION}

The advantages of microcomputer control are readily apparent: (1) Only one observer-operator is required, since all the functions of the second operator are assumed by the computer. Computerization cost, approximately $\$ 4,500$, is quickly recovered because of reduced labor requirements. (2) The observer-operator is not obliged to communicate judgments to a second operator; therefore, the observer-operator's entire attention can be directed toward the infant. (3) Recording errors that result from miscommunication between the observer and the second operator can no longer occur. (4) Since the computerized PL provides for a highly standardized test procedure, less technical training and understanding of methodology is required for one to administer the test and derive its results. (5) Any distracting influence upon the observeroperator or the infant engendered by the presence of a second operator in the test room is eliminated. (6) Stimulus gratings are automatically and quickly assigned to the left and right screens in a constrained random fashion. (7) All test results and parameters are rapidly and consistently calculated. (8) Results are directly stored on diskettes for later retrieval and analysis. (9) Whenever the observer-operator deems it appropriate, the apparatus can be reconverted to a standard twooperator manual system. (10) Alternate psychophysical methods can be implemented with the computer-controlled system.

The PL test in a clinical setting provides a quantitative measure of visual acuity in preverbal patients. Such a measure permits comparison with either established or establishable acuity age norms. It also permits quantitative comparisons between the acuities of the two eyes of the same patient. Both advantages are seen in the work of Catalano et al. (1987). Computerization of the PL provides these 
same advantages with many improvements, including a higher degree of standardization of the test, considerable reduction in the cost of technician time, the near elimination of several sources of test bias, and the provision of objective measures of potential test failure.

\section{REFERENCES}

BIRCH, E. (1985). Infant interocular acuity differences and binocular vision. Vision Research, 25, 571-576.

Catalano, R., Simon, J. W., Jenkins, P. L., \& Kandel, G. L. (1987). Preferential looking as a guide for amblyopia therapy in monocular infantile cataracts. Joumal of Pediatric Ophthalmology \& Strabismus, 24, 56-63.

FanTZ, R. L. (1958). Pattern vision in young infants. Psychological Record, 8, 43-47.

Gwiazda, J., BrILl, S., Mohindra, I., \& Held, R. (1978). Infant visual acuity and its meridional variation. Vision Research, 18, 1557-1564.

Gwiazda, J., Brill, S., Mohindra, I., \& Held, R. (1980). Preferential looking acuity in infants from two to fifty-eight weeks of age. American Joumal of Optometry \& Physiological Optics, 57, 428-432.
Gwiazda, J., Wolfe, J., Brill, S., Mohindra, I., \& Held, R. (1980). Quick assessment of preferential looking acuity in infants. American Journal of Optometry \& Physiological Optics, 57, 420-427.

Jenkins, P. L., Simon, J. W., Kandel, G. L., \& Forester, T. (1985). A simple grating visual acuity test for impaired children. American Journal of Ophthalmology, 99, 652-658.

LEvITT, H. (1970). Transformed up-down methods in psychoacoustics. Journal of the Acoustical Society of America, 49, 467-477.

Rose, R. M., Teller, D. Y., \& Rendleman, P. (1970). Statistical properties of staircase estimates. Perception \& Psychophysics, 8 , 199-204.

Teller, D. Y., Morse, R., Barton, R., \& Regal, D. (1974). Visual acquity for vertical and diagonal gratings in human infants. Vision Research, 14, 1433-1439.

\section{NOTE}

1. All monochrome grating transparencies were obtained from $\mathrm{J}$. Bauer, Psychology Department, Massachusetts Institute of Technology, Cambridge, MA.

(Manuscript received January 13, 1989; revision accepted for publication March 31, 1989.) 